\title{
Hyperactivated Wnt- $\beta$-catenin signaling in the absence of sFRP1 and sFRP5 disrupts trophoblast differentiation through repression of $\mathrm{Ascl} 2$
}

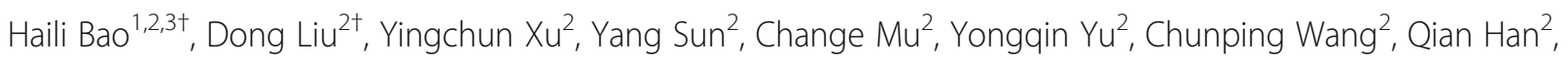
Sanmei Liu' ${ }^{2}$, Han Cai ${ }^{1,2}$, Fan Liü ${ }^{2}$, Shuangbo Kong ${ }^{1,2}$, Wenbo Deng ${ }^{1,2}$, Bin Cao ${ }^{1,2}$, Haibin Wang ${ }^{1,2^{*}}$, Qiang Wang ${ }^{3,4^{*}}$ and Jinhua Lu $\mathrm{Lu}^{1,2^{*}}$

\begin{abstract}
Background: Wht signaling is a critical determinant for the maintenance and differentiation of stem/progenitor cells, including trophoblast stem cells during placental development. Hyperactivation of Wnt signaling has been shown to be associated with human trophoblast diseases. However, little is known about the impact and underlying mechanisms of excessive Wnt signaling during placental trophoblast development.

Results: In the present work, we observed that two inhibitors of Wnt signaling, secreted frizzled-related proteins 1 and 5 (Sfrp1 and Sfrp5), are highly expressed in the extraembryonic trophoblast suggesting possible roles in early placental development. Sfrp1 and Sfrp5 double knockout mice exhibited disturbed trophoblast differentiation in the placental ectoplacental cone (EPC), which contains the precursors of trophoblast giant cells (TGCS) and spongiotrophoblast cells. In addition, we employed mouse models expressing a truncated $\beta$-catenin with exon 3 deletion globally and trophoblast-specifically, as well as trophoblast stem cell lines, and unraveled that hyperactivation of canonical Wnt pathway exhausted the trophoblast precursor cells in the EPC, resulting in the overabundance of giant cells at the expense of spongiotrophoblast cells. Further examination uncovered that hyperactivation of canonical Wnt pathway disturbed trophoblast differentiation in the EPC via repressing Ascl2 expression.

Conclusions: Our investigations provide new insights that the homeostasis of canonical Wnt- $\beta$-catenin signaling is essential for EPC trophoblast differentiation during placental development, which is of high clinical relevance, since aberrant Wnt signaling is often associated with trophoblast-related diseases.
\end{abstract}

Keywords: Sfrp1 and Sfrp5, Hyperactivation, Canonical Wnt pathway, Trophoblast, Ascl2

\footnotetext{
*Correspondence: haibin.wang@vip.163.com; wqiang2000@126.com; jinhua888@126.com

${ }^{+}$Haili Bao and Dong Liu contributed equally to this work.

${ }^{1}$ Reproductive Medical Center, The First Affiliated Hospital of Xiamen

University, Xiamen 361003, Fujian, People's Republic of China

${ }^{3}$ State Key Laboratory of Stem Cell and Reproductive Biology, Institute of

Zoology, Chinese Academy of Sciences, Beijing 100101, People's Republic of

China

Full list of author information is available at the end of the article
}

(c) The Author(s). 2020 Open Access This article is licensed under a Creative Commons Attribution 4.0 International License, which permits use, sharing, adaptation, distribution and reproduction in any medium or format, as long as you give appropriate credit to the original author(s) and the source, provide a link to the Creative Commons licence, and indicate if changes were made. The images or other third party material in this article are included in the article's Creative Commons licence, unless indicated otherwise in a credit line to the material. If material is not included in the article's Creative Commons licence and your intended use is not permitted by statutory regulation or exceeds the permitted use, you will need to obtain permission directly from the copyright holder. To view a copy of this licence, visit http://creativecommons.org/licenses/by/4.0/ The Creative Commons Public Domain Dedication waiver (http://creativecommons.org/publicdomain/zero/1.0/) applies to the data made available in this article, unless otherwise stated in a credit line to the data. 


\section{Background}

The placenta, forming the fetal-maternal interface, is essential for the survival and growth of the fetus in eutherian mammals $[1,2]$. In mice, a mature placenta consists of three trophoblast layers: the outermost giant cell layer, the intermediate spongiotrophoblast layer, and the innermost labyrinth layer. The trophoblast cells of the placenta arise from the outer trophectoderm of the blastocyst. After implantation (E4.5), while the mural trophectoderm cells stop dividing but keep endoreduplication to form the primary trophoblast giant cells invading into the uterus, the polar trophectoderm cells maintain proliferating and form diploid extraembryonic ectoderm (ExE) and ectoplacental cone (EPC), from the outer regions of which more TGCs form. Subsequently, the ExE form the chorion layer which gives rise to the labyrinth including two syncytiotrophoblast layers, while the EPC develops into the spongiotrophoblast layer [1]. The balanced differentiation of various trophoblast cell types is a prerequisite for normal placentation [3, 4]. The achaete-scute complex homolog-like 2 ( Ascl2, also Mash2), one member of the basic helix-loop-helix (bHLH) family, has been shown to play a critical role during EPC development. Ascl2 is located in the EPC and diminishes as trophoblast cells differentiate into TGCs. Ablation of Ascl2 led to embryonic lethality owing to defective spongiotrophoblast formation [5]. Moreover, ASCL2 is reported to be expressed in human freshly isolated cytotrophoblasts [6] and proximal column extravillous trophoblasts [7], corresponding to the EPC and/or spongiotrophoblast layer in mice. However, the signals that affect EPC trophoblast differentiation and how they interact with $A s c l 2$ remain largely unknown.

Previous studies have provided evidence that Wnt signaling is a critical player during placental development [8-15]. Wnt7b null mice died at mid-gestation stage due to defective chorioallantoic fusion [10]. Targeted disruption of Wnt2 caused impaired labyrinth [11]. Fzd5 mutation was reported to disturb placental labyrinth development [12], and our previous work further demonstrated the necessity of canonical Wnt2-Fzd5-Gcm1 (Glial cells missing-1) signaling for chorioallantoic branching and trophoblast syncytialization during placentation [9]. Recently, Wnt signal was shown to be essential for the derivation and maintenance of human trophoblast stem cells (HTSCs) and organoids [16-18]. Although Wnt signaling is essential for normal placental development, hyperactivation of Wnt signaling are often observed in human trophoblast-related diseases such as complete hydatidiform moles and choriocarcinoma [8, 19]. However, whether and how excessive Wnt signaling affects EPC trophoblast differentiation remains largely unclear.
In the present work, we employed a variety of genetic mouse models and cultured trophoblast cells to demonstrate that hyperactivation of canonical Wnt pathway leads to overabundance of TGCs at the expense of spongiotrophoblast cells, via repressing Ascl2 during EPC trophoblast differentiation, providing direct genetic evidence for the crucial role of suitable canonical Wnt signaling during placental trophoblast differentiation.

\section{Results}

Sfrp1 and Sfrp5 deficiency leads to excessive TGC differentiation and compromised placental development

Secreted frizzled-related proteins (SFRPs), containing a cysteine-rich domain (CRD) that is 30 to $50 \%$ similar in sequence to that of the frizzled protein while lacking the transmembrane domain, serve mainly as extracellular inhibitors of Wnt signaling by directly blocking the interactions between the Wnt and Frizzled receptors [20]. Sequence comparison and phylogenetic analysis reveal that SFRP1, SFRP2, and SFRP5 are closely related [21]. To define the potential functions of these SFRPs during placentation, we firstly examine the expression profile of Sfrp1, Sfrp2, and Sfrp5 during early placental development. Through whole-mount in situ hybridization, we observed that both Sfrp1 and Sfrp5 were expressed in the extraembryonic tissues at E7.5 and E8.5, except Sfrp2 (Additional file 1: Fig. S1A). Further examination of the placental sections at E7.5-E9.5 showed that the expression of Sfrp1 and Sfrp5 was abundant in extraembryonic trophoblast, including trophoblast cells in the chorion and EPC, as well as the subsequent spongiotrophoblast layer (Additional file 1: Fig. S1B). These findings suggest the potential roles of Sfrp1 and Sfrp5 during early placental development.

To assess the physiological relevance of Sfrp1 and Sfrp5 during placental development, we examined the pregnancy outcome of Sfrp1 and Sfrp5 double knockout (dKO) females crossed with $\mathrm{dKO}$ males, and observed that the average litter size was significantly reduced, compared with that of the wildtype (WT) intercrosses $\left(4.5 \pm 0.3\right.$ vs $7.0 \pm 0.4,{ }^{*} P<0.05$, Fig. 1a). We subsequently analyzed the stage-specific effect of Sfrp1 and Sfrp5 during pregnancy. As illustrated in Fig. 1b, normal embryo implantation exhibited by blue bands was observed in both $\mathrm{dKO}$ and WT mice at E4.5, and the average number of implantation sites in $\mathrm{dKO}$ mice was comparable to that of the WT mice $(6.2 \pm 0.5$ vs $7.4 \pm$ 0.4). Moreover, Sfrp1 and Sfrp5 deficiency did not hamper uterine decidualization, displayed by the normal weight and size of implantation sites at E7.5 (21.84 \pm $0.47 \mathrm{mg}$ vs $21.01 \pm 0.94 \mathrm{mg}$, Fig. 1c). However, increased rate of embryo degeneration was observed in $\mathrm{dKO}$ mice at E11.5, $\left(32.4 \pm 4.1 \%\right.$ vs $6.0 \pm 4.3 \%,{ }^{*} P<0.05$, Fig. $\left.1 d\right)$. Further histological examination and immunostaining 


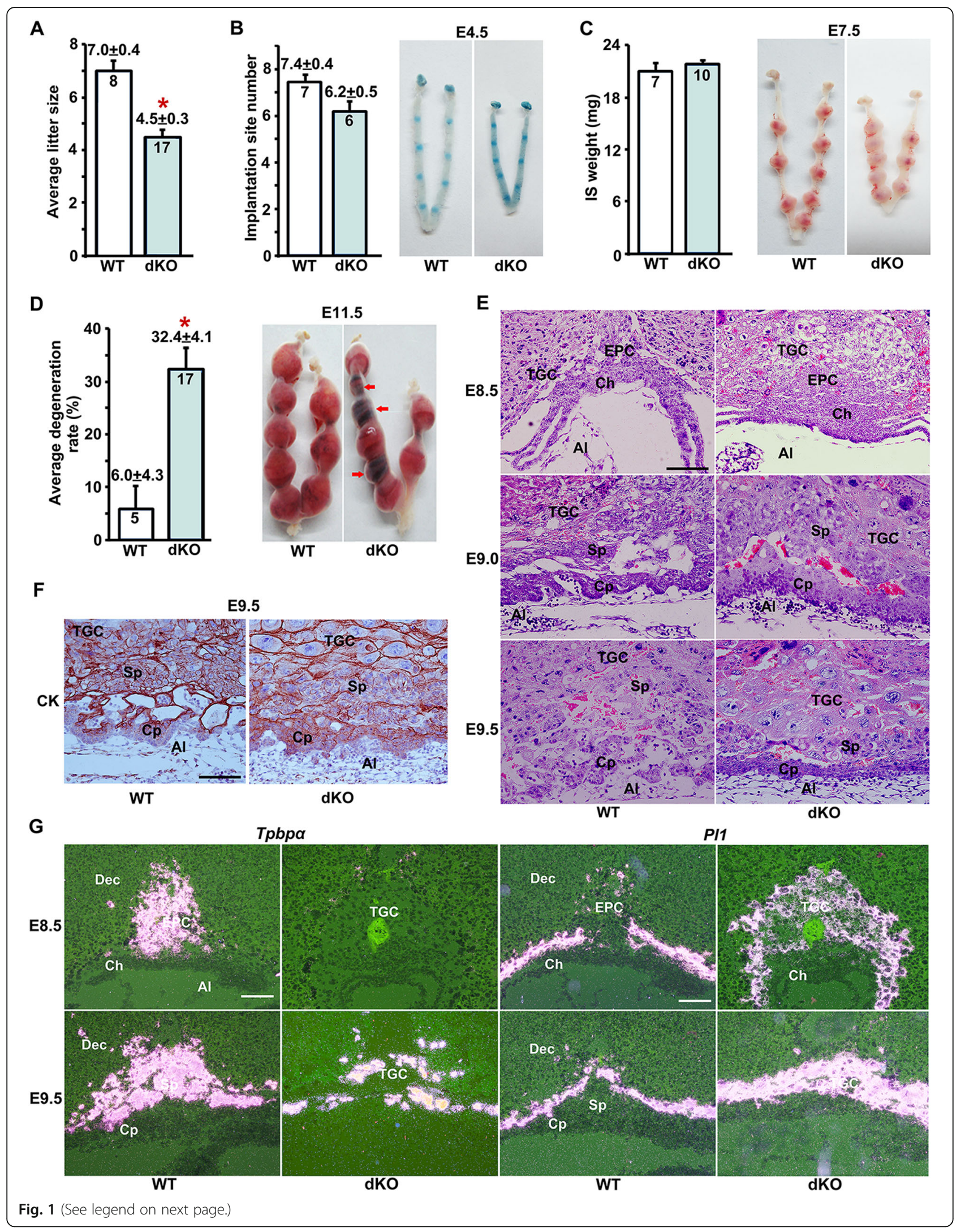


(See figure on previous page.)

Fig. 1 Sfrp1 and Sfrp5 deficiency leads to excessive TGC differentiation and compromised placental development. a Average litter sizes of WT as well as Sfrp 1 and Sfrp5 double knockout (dKO) mice. ${ }^{*} P<0.05$. b Average number of implantation sites and implantation status of WT and dKO mice at E4.5. c Average weight of implantation sites and implantation status of WT and dKO mice at E7.5. $\mathbf{d}$ Average embryo degeneration rate and representative uteri of WT and dKO mice at E11.5. Arrows indicated degenerated embryos. ${ }^{*} P<0.05$. e Hematoxylin and eosin (HE) staining of E8.5-E9.5 placental sections of WT and dKO mice. $\mathbf{f}$ Immunostaining analysis of cytokeratin (CK) on E9.5 placental sections of WT and dKO mice. $\mathbf{g}$ In situ hybridization analysis of the expression of Tpbpa and P/1 in the placentas of WT and dKO mice, at E8.5 and E9.5. In a-d, numbers within the bars indicated the number of pregnant mice examined. Images in $\mathbf{e}-\mathbf{g}$ are representatives of at least three independent experiments. Al, allantois; Ch, chorion; Cp, chorionic plate; Sp, spongiotrophoblast; TGC, trophoblast giant cell. Scale bar, $100 \mu \mathrm{m}$

analysis of cytokeratin (CK) marking placental trophoblast cells revealed impaired differentiation of extraembryonic trophoblast lineage with expanded TGCs and reduced spongiotrophoblast layer as well as compact chorion, at E8.5-E9.5 (Fig. 1e, f, and Additional file 1: Fig. S2A). In addition, we performed in situ hybridization to examine the expression of marker genes placental lactogen I (Pl1), specifically expressed in TGCs, and trophoblast-specific protein $\alpha$ (Tpbp $\alpha)$, marking EPC and/or spongiotrophoblast cells. While Tpbpa ${ }^{+}$ trophoblast cells were decreased, Pl1-expressing trophoblast cells were significantly increased in dKO mice (Fig. 1g). These data reveal that $S f r p 1$ and Sfrp5 deletion disturbs EPC trophoblast differentiation with excessive TGCs during early placentation.

\section{Sfrp 1 and Sfrp 5 deletion renders hyperactivation of canonical Wnt signaling and disturbs trophoblast differentiation in the EPC}

Since Sfrp1 and Sfrp5 regulate both the canonical and noncanonical Wnt pathway, we firstly detected the activity of canonical Wnt pathway. We observed that the nuclear localization of active- $\beta$-catenin, an indicator of canonical Wnt signaling activity, was increased significantly in the EPC and TGCs of dKO placentas at E8.5 (Fig. 2a and Additional file 1: Fig. S2B), which was further confirmed by western blot analysis (Fig. 2b). These results suggest that the absence of Sfrp1 and Sfrp5 enhanced the activity of canonical Wnt signaling. Considering the phenotype of reduced EPC trophoblast cells and increased number of TGC in $\mathrm{dKO}$ placentas, we
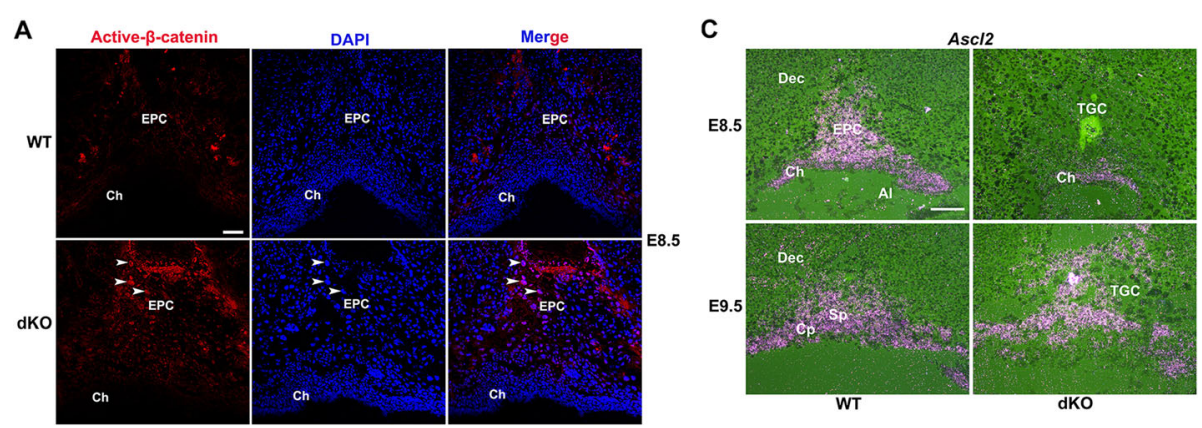

B

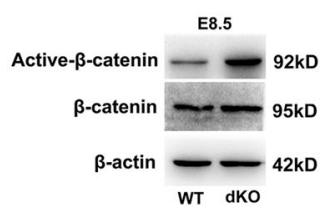

D

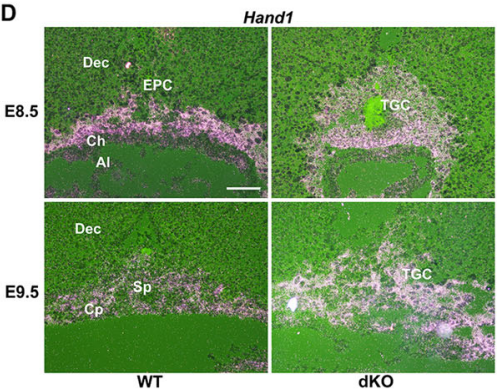

Fig. 2 Sfrp1 and Sfrp5 deletion renders hyperactivation of canonical Wht signaling and disturbs EPC trophoblast differentiation. a, b Immunostaining and western blot analysis of active- $\beta$-catenin in WT and dKO placentas at E8.5. Cy3-labeled $\beta$-catenin in red, DAPI-labeled nuclei in blue. Arrowheads indicated trophoblast cells with nuclear localization of active- $\beta$-catenin. $\beta$-actin served as the internal control. $\mathbf{c}, \mathbf{d}$ The expression of Ascl2 and Hand1 was revealed by in situ hybridization in the WT and dKO placentas at E8.5 and E9.5. Images in a, c, and $\mathbf{d}$ are representatives of at least three independent experiments. Al, allantois; Dec, decidua; EPC, ectoplacental cone; Ch, chorion; Cp, chorionic plate; Sp, spongiotrophoblast; TGC, trophoblast giant cell. Scale bar, $100 \mu \mathrm{m}$ 
examined the expression of the key genes essential for trophoblast cell differentiation in the EPC. The genes Ascl 2 and Hand1 (heart and neural crest derivatives expressed transcript 1), encode two transcription factors of the basic helix-loop-helix (bHLH) family. Ascl2 is required to maintain the EPC progenitor population, determines the specification of Tpbp $\alpha$-positive trophoblast cells, and inhibits Pl1-expressing giant cell differentiation, while Hand 1 has opposing roles and promotes Pl1-expressing TGC differentiation [22]. Ablation of Ascl 2 or Hand1 in mice both lead to embryo lethality owing to defective placental development [5, 23, 24]. By in situ hybridization, we found that while $A s c l 2$-positive trophoblast cells were decreased (Fig. 2c), the number of Hand1-expression TGCs were increased significantly (Fig. 2d), in dKO mice at E8.5 and E9.5, similar to that of Ascl2 mutant mice [5]. These findings illustrate that loss of Sfrp1 and Sfrp5 increases the canonical Wnt signaling activity which might disturb the differentiation of trophoblast progenitors in the EPC.

\section{Global stabilization of $\beta$-catenin leads to impaired placental development with excessive TGC differentiation} Since Sfrp1 and Sfrp5 deletion led to exaggerated canonical Wnt signaling activity, to further define whether the hyperactivation of canonical Wnt signaling disturbed EPC trophoblast differentiation, we introduced the $C t n n b 1^{f(E X 3) / f(E X 3)}$ mouse model, in which the exon 3 of $\beta$-catenin gene is flanked by loxp sites, and Cre recombinase-mediated excision would give rise to the expression of a stabilized, constitutively active form of $\beta$ catenin resistant to degradation by the GSK3 $\beta$-mediated proteasome pathway [25]. Through crossing the
Ctnnb $1^{f(E X 3) / f(E X 3)}$ male with Prm-cre (functioning in adult testis) transgenic female, Prm-cre; Ctnnb1 $1^{f(E X 3) /+}$ male mice were generated. When crossing WT females with Prm-cre; Ctnnb $1^{f(E X 3) /+}$ males, the $C t n n b 1^{\Delta /+}$ conceptus with stabilized $\beta$-catenin and hyperactivated canonical Wnt pathway were generated. In this breeding, the average litter size decreased significantly, and Ctnnb $1^{\Delta /+}$ pups failed to be born (Fig. 3a, b) and degenerated before E11.5 (Fig. 3c, d). Moreover, histological examination and immunostaining analysis of $\mathrm{CK}$ showed excessive TGCs in the Ctnnb1 $1^{\Delta /+}$ conceptus at E9.5 (Fig. 3e), with increased activity of Wnt signaling (Additional file 1: Fig. S3), similar to the phenotype of Sfrp1, $5 \mathrm{dKO}$ mice. Further examination indicated that the defects of the Ctnnb1 $1^{\Delta /+}$ conceptus appeared as early as E7.5 (Additional file 1: Fig. S4). These data demonstrate that stabilized $\beta$-catenin disturbs placental development with excessive TGC differentiation.

\section{Trophoblast-specific stabilization of $\beta$-catenin impairs EPC trophoblast differentiation with excessive TGC differentiation}

To further verify the contributions of trophoblastspecifically stabilized $\beta$-catenin protein, we generated the CYP19-cre; Ctnnb1 $1^{f(E X 3) /+}$ mouse model, in which $\beta$ catenin protein is dominant-stabilized specifically in extraembryonic trophoblast cells. When CYP19-cre females were crossed with Ctnnb $f^{f(E X 3) /+}$ males, the average litter size was decreased significantly, and the CYP19-cre; Ctnnb1 $1^{f(E X 3) /+}$ offspring failed to be born (Fig. 4a, b) and degenerated before E11.5 (Fig. 4c-e). Histological analysis and immunostaining analysis of CK exhibited abnormal extraembryonic tissues with
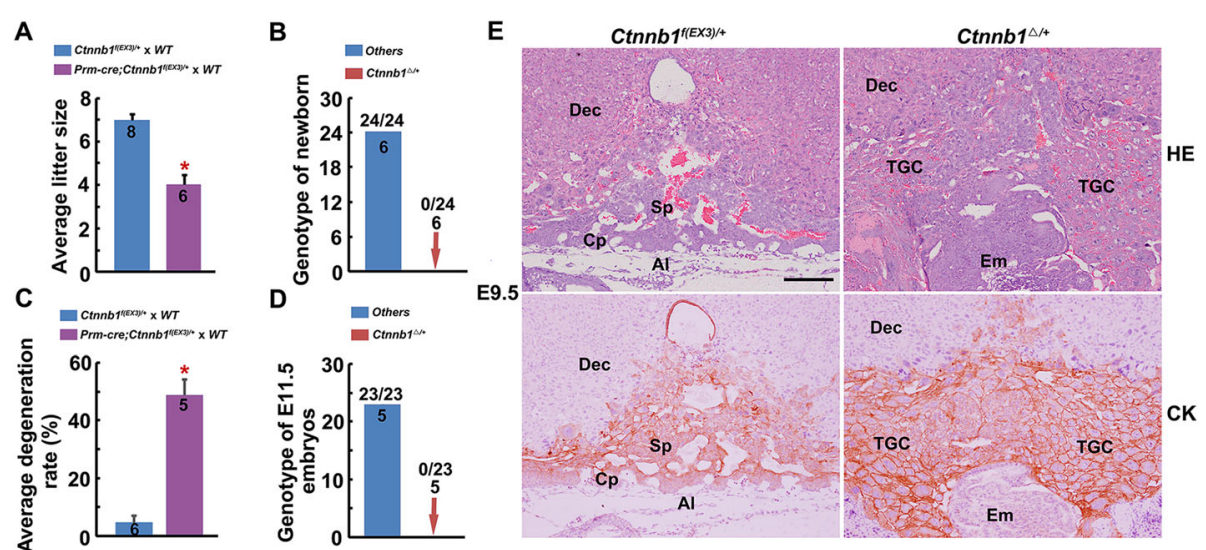

Fig. 3 Global stabilization of $\beta$-catenin leads to impaired placental development with excessive TGC differentiation. a Average litter sizes of WT females mated with $C$ tnnb $1^{f(E X 3) /+}$ and Prm-cre; $C$ tnnb $1^{f([X 3) /+}$ males, respectively. ${ }^{*} P<0.05$. b Genotyping of the newborns from WT females mated

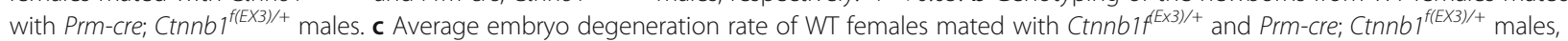
respectively, at E11.5. ${ }^{*} P<0.05$. d Genotyping of the remaining survived embryos from WT female mated with Prm-cre; $C \operatorname{Ctnnb} 1^{f(E \times 3) /+}$ male mice at

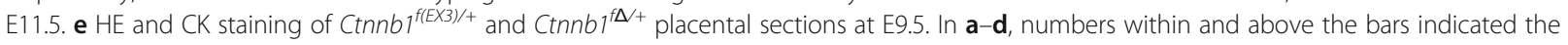
number of pregnant mice and embryos examined, respectively. Images in e are representatives of at least three independent experiments. Al, allantois; Dec, decidua; Cp, chorionic plate; Em, embryo; Sp, spongiotrophoblast; TGC, trophoblast giant cell. Scale bar, $100 \mu \mathrm{m}$ 


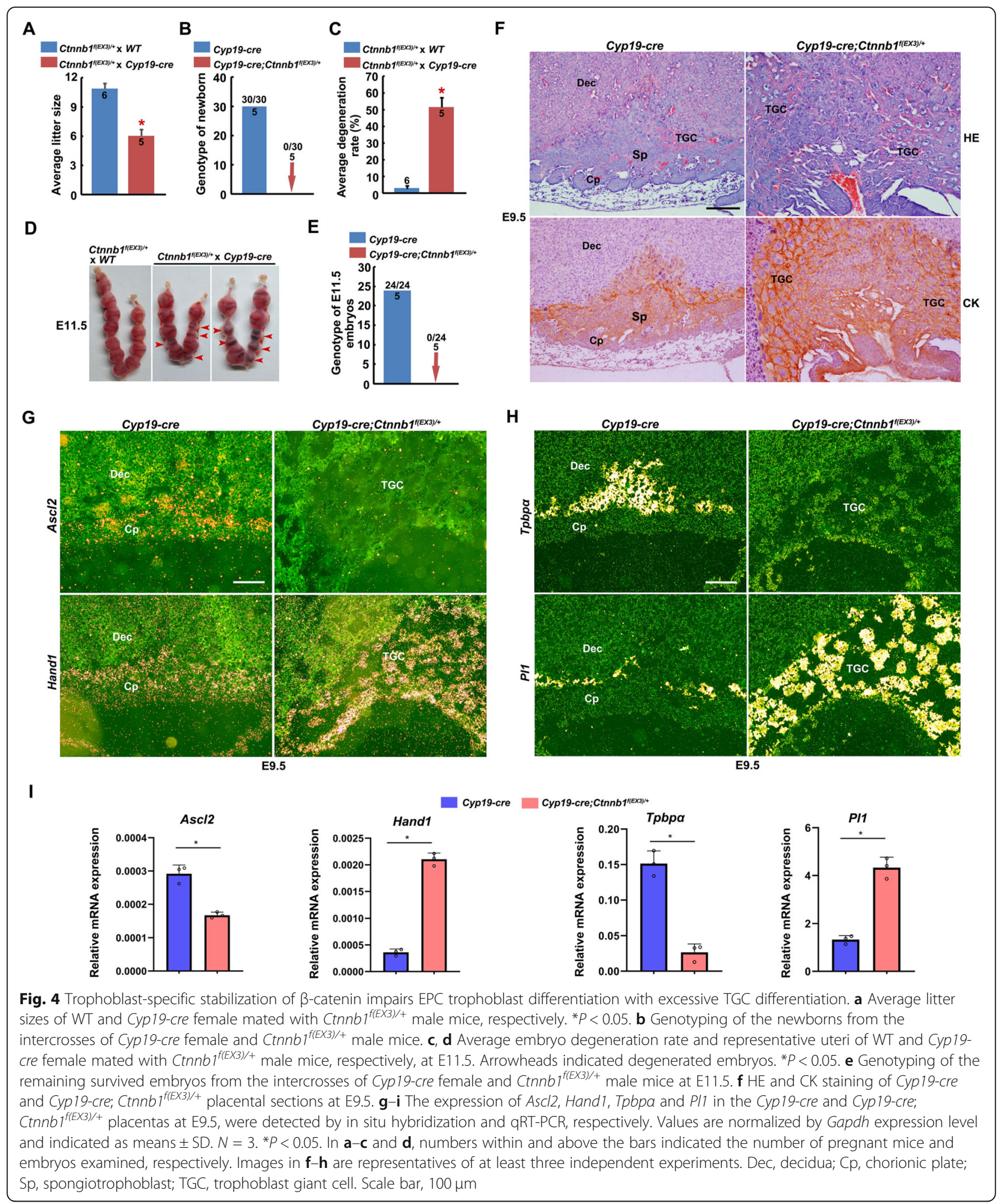

excessive TGCs differentiation in CYP19-cre; Ctnnb $f^{f(E X 3) /+}$ conceptus (Fig. 4f), which might be responsible for the embryo degeneration at mid-gestation and recapitulated the phenotypes of the Sfrp1, $5 \mathrm{dKO}$ and $C \operatorname{tnn} b 1^{\Delta /+}$ mice. Moreover, while the number of Ascl2- and Tpbpo-positive trophoblast cells decreased, Hand1- and Pl1-expressing TGCs were increased significantly in the CYP19-cre; Ctnnb1 $1^{f(E X 3) /+}$ placentas at E9.5 
(Fig. 4g, h). Quantitative RT-PCR (qRT-PCR) analysis also showed decreased expression of Ascl2 and Tpbpa and increased expression of Hand1 and Pl1 in CYP19cre; $C \operatorname{tnn} b 1^{f(E X 3) /+}$ placentas (Fig. $\left.4 \mathrm{i}\right)$. These findings suggest that trophoblast cells in the EPC might differentiate excessively into TGCs in the presence of stabilized $\beta$ catenin. In addition, we observed increased intensity of nucleus-localized active- $\beta$-catenin in trophoblast cells of the EPC and TGCs of CYP19-cre; Ctnnb $1^{f(E X 3) /+}$ conceptus (Additional file 1: Fig. S5), consistent with the findings in the Sfrp1 and Sfrp5 mutant mice. In summary, trophoblast-specific stabilization of $\beta$-catenin induced hyperactivation of canonical Wnt pathway which impairs EPC trophoblast differentiation.

\section{Hyperactivation of canonical Wnt signaling disturbs EPC} trophoblast differentiation via repressing $A s c / 2$ expression To dissect the underlying mechanism by which hyperactivated canonical Wnt signaling influences trophoblast differentiation, we preformed RNA-seq on CYP19-cre and CYP19-cre; Ctnnb $1^{f(E X 3) /+}$ placentas at E8.5. 209 upregulated genes and 548 downregulated genes were observed in the CYP19-cre; Ctnnb1 $1^{f(E X 3) /+}$ placentas (fold change $>1.5, P$ value $<0.05$, Fig. 5 a, Additional file 2 : Table S2). Gene ontology (GO) enrichment analysis revealed that the upregulated genes, including $\operatorname{Prl} 2 c 2$, $\operatorname{Prl3d1}$, and Prl7b1, were related to the female reproduction and the regulation of lactation (Fig. 5b), possibly due to the increased number of TGCs expressing and/or secreting these molecules; the downregulated genes were related to cell cycle and cell division (Fig. 5c and Additional file 1: Fig. S6), which might be resulted from the excessive differentiation of TGCs without nuclear and cytoplasmic division. Moreover, the expression of genes located in different trophoblast cell types were examined in the CYP19-cre and CYP19-cre; Ctnnb $f^{f(E X 3) /+}$ placentas (Additional file 2: Table S3), and their expression patterns were consistent with the placental defects (Fig. 5d). In addition, we observed that several Wnt target genes, such as Vegfa, Mmp9, Myc, Ppard, and Gcm1, were upregulated in CYP19-cre; Ctnnb $f^{f(E X 3) /+}$ placentas (Additional file 2: Table S3), which was further confirmed by qRT-PCR analysis (Fig. 5e), indicating the enhanced activity of canonical Wnt signaling.

As a marker of progenitor trophoblast cells in the EPC, Ascl2 also functions as an important transcription factor essential for the maintenance of the EPC, since Ascl2 deletion results in increased number of giant cells at the expense of the EPC layer [5], similar to the phenotypes in our present study. Moreover, Ascl2 was reported to be a target of canonical Wnt signaling in intestinal neoplasia and intestinal stem cells [26, 27]. Indeed, according to our RNA-Seq data, Ascl2 expression was decreased in the placenta with hyperactivated canonical Wnt signaling, which was further confirmed by qRTPCR analysis (Fig. 5f). Based on the above observations, we speculated that Ascl2 might be a target of canonical Wnt signaling during EPC trophoblast differentiation.

\section{Excessive canonical Wnt signaling activity restrains spongiotrophoblast differentiation through suppressing Ascl2 in cultured trophoblast cells}

In order to verify the hypothesis that hyperactivation of canonical Wnt signaling disturbs the EPC differentiation via repressing Ascl2 expression, we further employed in vitro cultured trophoblast stem (TS) cell lines, which undergo differentiation in the absence of FGF4 and mitomycin C-treated mouse embryonic fibroblastconditioned medium (FCM) [28]. Compared with WT TS cells, Sfrp 1 and Sfrp 5 dKO TS cells showed decreased expression of Ascl2 and Tpbpa during differentiation (Fig. 6a, c), consistent with the in vivo observations. Moreover, the level of active- $\beta$-catenin protein (Fig. $6 \mathrm{~b}$ ) and the nuclear localization of active- $\beta$-catenin (Fig. $6 \mathrm{c}$ ) were increased significantly in the absence of Sfrp1 and Sfrp5. TOP-Flash assay further confirmed hyperactivated $\beta$-catenin transcription activity in Sfrp1 and Sfrp 5 dKO TS cells (Additional file 1: Fig. S7A). To confirm the contributions of hyperactivated canonical Wnt pathway to disturbed trophoblast cell differentiation, we employed CHIR99021 (CHIR), the agonist of canonical Wnt pathway [29]. After treatment with CHIR, the nuclear localization of $\beta$-catenin was enhanced (Fig. 6d), and the levels of active- $\beta$-catenin protein were increased remarkably (Fig. 6e). Meanwhile, the expression level of Ascl2 and Tpbp $\alpha$ were decreased significantly (Fig. 6d, f). On the contrary, Sfrp1 and Sfrp5 dKO TS cells treated with XAV939, an inhibitor of Wnt pathway, or with Ascl2 overexpression exhibited increased expression of Tpbp $\alpha$ and decreased Pl1 expression (Additional file 1: Fig. S7B and C). These findings demonstrate that excessive canonical Wnt signaling represses Ascl2 expression which is responsible for the disturbed trophoblast differentiation.

Generally, upon the activation of canonical Wnt pathway, stabilized $\beta$-catenin translocate into the nucleus, where it serves as a coactivator of the TCF/LEF DNA binding factors, to activate the transcription of target genes. In colorectal cancer cells, with the assistance of a neighboring cis-acting lincRNA, TCF $4 / \beta$-catenin complex was recruited to the $A s c l 2$ enhancer immediately downstream of the Ascl2 locus to drive high-level Ascl2 expression [30]. Moreover, $\beta$-catenin bound to enhancers and direct enhancer-promoter looping at mesendodermal (ME) lineage genes in human embryonic stem cells (hESC) [31]. To testify how canonical Wnt signaling regulate $A s c l 2$ expression, we performed 


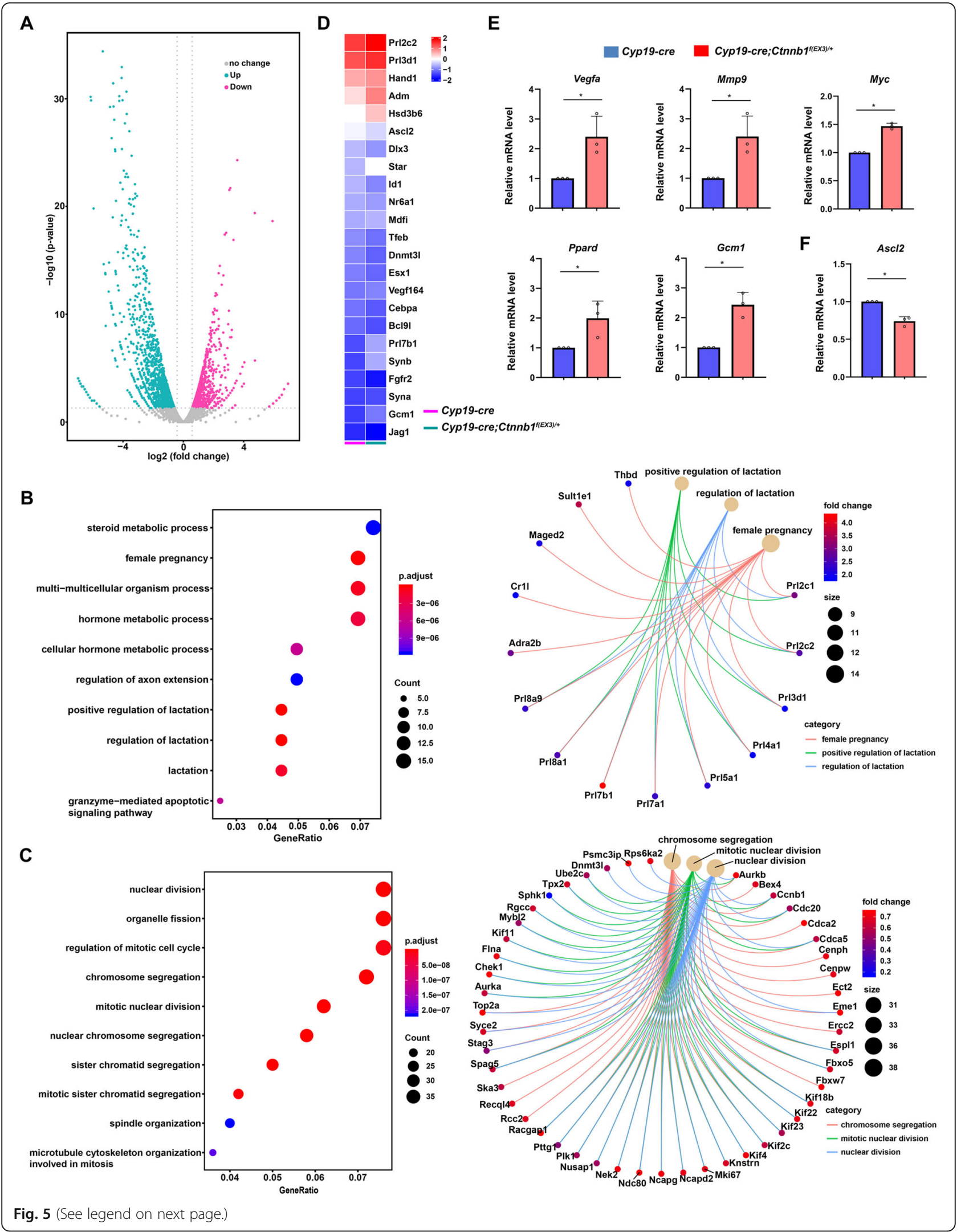


(See figure on previous page.)

Fig. 5 Hyperactivation of canonical Wnt signaling disturbs EPC trophoblast differentiation via repressing Ascl2 expression. a Volcano plot displaying differentially expressed genes (DEG) between the Cyp19-cre and Cyp19-cre; Ctnnb $1^{f(E X 3) /+}$ placentas (fold change $>1.5, P$ value $<0.05$ ). $\mathbf{b}$,

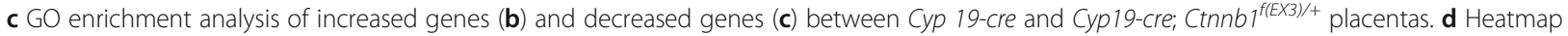
showing the expression of trophoblast-located genes in the Cyp19-cre and Cyp19-cre; Ctnnb $1^{f([X 3) /+}$ placentas. e, $\mathbf{f}$ The expression of Wnt target genes and AsCl2 was confirmed by qRT-PCR. Values are normalized by Gapdh expression level and indicated as means \pm SD. $N=3$. ${ }^{*} P<0.05$

chromatin immunoprecipitation and sequencing (ChIPSeq) to assess the genome-wide occupancy of $\beta$-catenin in differentiated TS cells in the presence of CHIR or not. As expected, $\beta$-catenin occupied the promoter regions of the known Wnt target genes, such as Axin2 and Gcm1 (Fig. 6g). However, $\beta$-catenin exhibited enhanced enrichment at a site about $20 \mathrm{~kb}$ upstream of the $A s c l 2$ promoter in the presence of CHIR (Fig. 6h), which was confirmed by ChIP-qPCR analysis (Fig. 6i). These data suggest that hyperactivation of canonical Wnt signaling might repress Ascl 2 expression through $\beta$-catenin binding to the remote upstream regions of the Ascl2 locus during trophoblast differentiation.

\section{Discussion}

The canonical Wnt- $\beta$-catenin signaling is essential for a variety of biological processes, including embryogenesis, stem cell maintenance and differentiation, even the cell self-renewal of cancer stem cells [32-34]. Loss or hyperactivation of canonical Wnt signaling often leads to disturbed developmental processes or even diseases [32, 35]. We provide herein genetic and molecular evidence that hyperactivation of canonical Wnt signaling disturbs trophoblast differentiation in the EPC via repression Ascl2 expression during placental development. These findings highlight the necessity of suitable Wnt signaling during trophoblast differentiation.

Wnt signaling has been reported to regulate the proliferation and differentiation of stem and progenitor cells, during the processes of both embryonic development and adult tissue homeostasis [34, 36], and aberrant Wnt signaling underlies various human diseases [32]. Human colon cancer with $A P C$ mutation, rendering inappropriate $\beta$-catenin stabilization and hyperactivated canonical Wnt pathway [37], exhibited a hereditary cancer syndrome named familiar adenomatous polyposis (FAP) $[38,39]$. Excessive activation of Wnt signaling led to pathological bone deposition and hardening [32]. As to placental development, previous studies showed that the deletion of several members of Wnt signaling pathway impaired placental development [9-15]. Moreover, Fzd5-mediated canonical Wnt signaling has been proven essential for trophoblast syncytialization in both human and mouse [9]. Recent findings demonstrate the necessity of Wnt signaling for the derivation and maintenance of hTSCs [17] and organoids [16, 18]. However, excessive Wnt signaling disturbs development and functions of placental trophoblast cells. In complete hydatidiform mole (CHM) placenta, elevated expression of nuclear $\beta$-catenin in the extravillous trophoblasts (EVT) were observed, indicating that aberrant hyperactivation of Wnt pathway contributes to abnormal invasive trophoblast differentiation and invasion [19]. Moreover, inactivation of negative regulators of Wnt signaling, such as APC and SFRP2, was found in choriocarcinoma cells, suggesting that higher activity of Wnt pathway is involved in the progress and function of trophoblast cancers $[40,41]$. On the basis of the findings, we wonder what would occur if the activity of Wnt signaling were elevated abnormally during placental development. In the present study, employing different genetic mouse models (Sfrp1 and Sfrp5 dKO mice, Prm-cre; Ctnnb1 $1^{f(E X 3) /+}$ global null mice, and CYP19-cre; Ctnnb1 $1^{f(E X 3) /+}$ trophoblast-specific null mice) that render hyperactivation of canonical Wnt- $\beta$-catenin pathway, we found that increased activity of canonical Wnt pathway promoted the excessive differentiation of TGCs via downregulating Ascl 2 expression. In addition, since canonical Wnt activity has been reported to promote trophoblast invasion [42], hyperactivation of canonical Wnt signaling in the differentiated TGCs might abnormally promote the invasive ability of these TGCs, leading to their excessive invasion into the maternal decidua, which may help explain the pathogenesis of human accrete pregnancy and worth further investigation.

In addition, as the inhibitors of Wnt pathway, loss of Sfrp1,5 induced hyperactivation of canonical Wnt signaling which impaired trophoblast differentiation, suggesting that the mechanism that keeps the activity of canonical Wnt signaling in a suitable state to maintain the niche for trophoblast differentiation and TGC development, exists in vivo during normal placentation. In order to maintain the niche, a complicated signaling network is needed to preserve the balance of trophoblast differentiation.

Ascl2 is essential for the maintenance of the EPC during early placental development in mice [5]. Ascl2 knockout and hypomorphic mouse model showed abnormal placentation with decreased EPC layer and/or spongiotrophoblast cell lineage as well as increased number of TGCs $[5,43]$. In the present study, we found the similar phenomenon when canonical Wnt signaling 


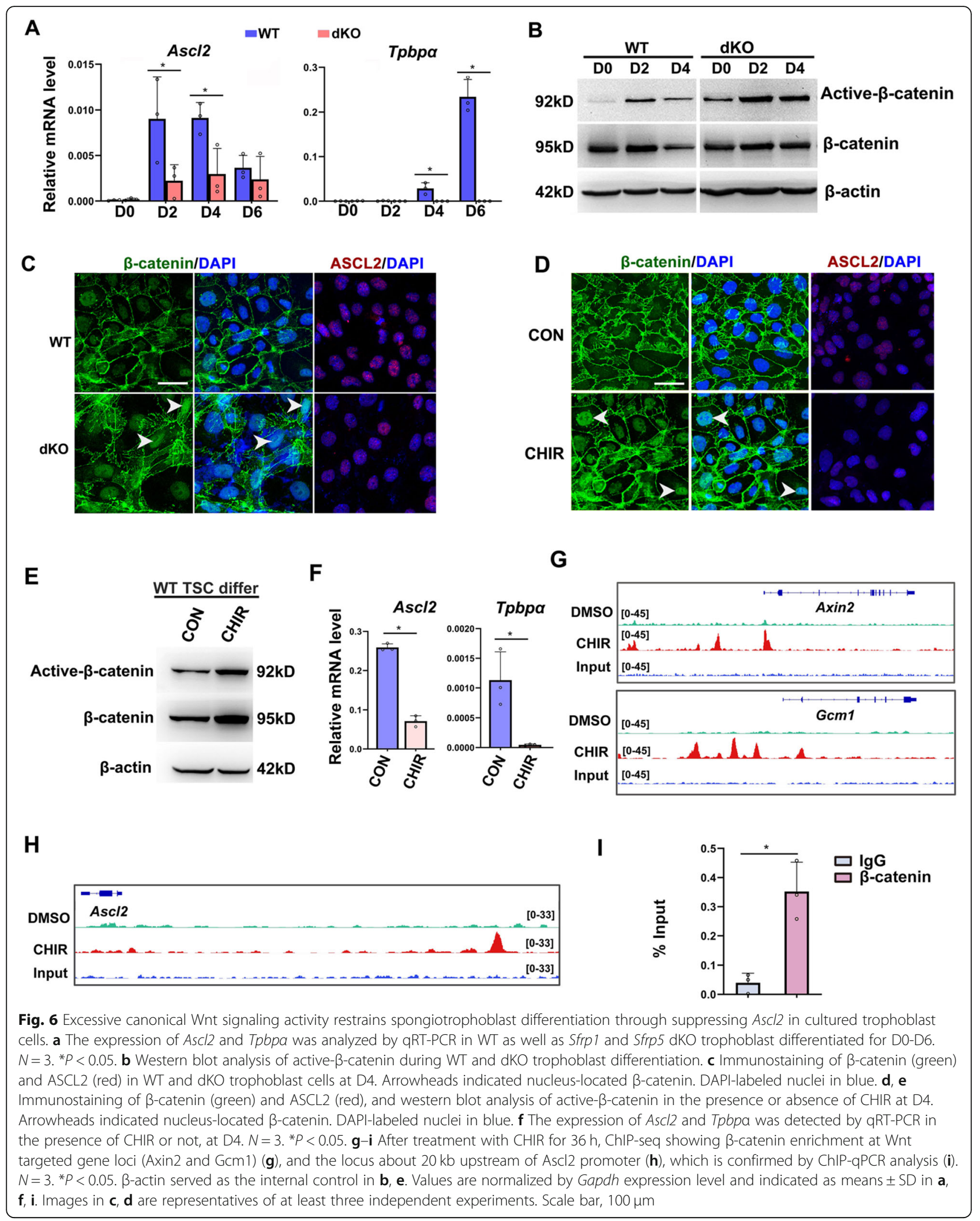


was hyperactivated and Ascl2 expression was repressed. However, little is known concerning how canonical Wnt signaling regulates $A s c l 2$ expression. In most cases, the canonical Wnt- $\beta$-catenin signaling stimulates the transcription of target genes, such as $c-M y c$ [44], Axin2 [45] and $\operatorname{Gcm} 1[9,15]$, through promoter binding by the transcriptional activator complex containing LEF/TCF and $\beta$ catenin. In the present work, we observed that $\beta$-catenin exhibited enhanced enrichment at the site about $20 \mathrm{~kb}$ upstream of Ascl2 locus upon CHIR treatment. However, TCF/LEF might be not required for $\beta$-catenin-mediated Ascl2 suppression (Additional file 1: Fig. S8). Since $\beta$ catenin has been reported to bind to the downstream or upstream enhancer to promote the expression of its target genes [30, 31], we speculated that excessive canonical Wnt signaling might regulate $A s c l 2$ expression in a similar manner, but to repress Ascl 2 expression. Moreover, recent findings have demonstrated that canonical Wnt pathway inhibits the expression of its target genes, including the tumor suppressor 15-prostaglandin dehydrogenase [46], RANKL [47] and E-cadherin [48]. However, the exact mechanism via which canonical Wnt signaling represses gene expression requires further exploration.

The chorionic development was also impaired, even though the expression of Gcm1, essential for chorionic trophoblast differentiation [49] and regulated by canonical Wnt signaling [9], was increased upon the hyperactivation of canonical Wnt pathway (Fig. 5e). One possibility is that while canonical Wnt signaling is essential for chorionic trophoblast differentiation, hyperactivation of canonical Wnt signaling which increases Gcm1 expression, is not necessarily beneficial for terminal differentiation of chorionic trophoblast. This could be explained by the fact that Gcm1 overexpression just induces a rapid arrest of trophoblast proliferation and restricts their differentiation fate towards syncytiotrophoblast cells, but is not sufficient to render cell shape changes or cell-cell fusion during subsequent terminal differentiation and syncytium formation. Another possibility is that the disturbed EPC differentiation with reduced $A s c l 2$ expression resulting from hyperactivated canonical Wnt signaling, led to defective chorionic trophoblast differentiation, since the mouse models with Ascl2 deletion [5] or reduced Ascl2 expression [50] also displayed disturbed chorionic/labyrinth development. Even though it has been shown that Ascl2-expressing trophoblast cells was not required for labyrinth development [51], this work is not imprecisely, since the wildtype trophoblast could provide the labyrinth layer with Ascl2-expressing trophoblast cells. In addition, the EPC/ spongiotrophoblast layer might functions as structural support for the chorionic/labyrinth development, since Ascl2-expressing trophoblast cells are also present in the chorion and subsequent labyrinth layer.

\section{Conclusions}

In summary, hyper-activation of the canonical Wnt signaling, achieved by either the deletion of Sfrp1,5 or the expression of a stabilized $\beta$-catenin globally and trophoblast-specifically, resulted in impaired EPC trophoblast differentiation and excessive TGC expansion. Further exploration using the trophoblast stem cell line uncovered that hyper-activated Wnt pathway led to the repression of $A s c l 2$, which is important for trophoblast differentiation. This study provides genetic and molecular evidence that appropriate canonical Wnt pathway is crucial for EPC trophoblast differentiation, which is of high clinical significance since there is a strong correlation between abnormal Wnt signaling and human trophoblast diseases [8].

\section{Materials and methods}

\section{Animals and tissue collection}

Ctnnb $f^{f(E X 3) / f(E X 3)}$ mice, Sfrp1 and Sfrp5 double mutant mice, and Cyp19-cre transgenic mice were generated as previously described [25, 52-54]. Prm-cre transgenic mice were obtained from Jackson Laboratory. Ctnnb $f^{f(E X 3) / f(E X 3)}$ mice were mated with Prm-cre mice to get mice with global hyperactivation of the canonical Wnt pathway. Ctnnb1 $1^{f(E X 3) / f(E X 3)}$ mice were mated with Cyp19-cre transgenic mice to get trophoblast-specific hyperactivation of canonical Wnt signaling. Eight-week females were mated with fertile males to induce pregnancy and the day when virginal plugs were seen was considered as embryonic day 0.5 (E0.5). Pregnant females were sacrificed and implantation sites were weighted, frozen, or fixed in $10 \%$ neutral buffered formalin. Tail genotyping of the embryos and newborns were determined by PCR.

\section{Histological analysis and immunostaining}

Histological and immunostaining analysis were performed as described previously [9]. In brief, dissected implantation sites were fixed in 10\% neutral buffered formalin at room temperature overnight. Tissues underwent dehydration using graded ethanol, vitrification by dimethylbenzene and were embedded in paraffin, and $5 \mu \mathrm{m}$ transverse sections were used for hematoxylin and eosin (H\&E) staining, immunohistochemistry (IHC), and immunofluorescence (IF). For frozen tissue, $10 \mu \mathrm{m}$ transverse sections were used for immunofluorescence (IF). Antibodies used for immunohistochemistry (IHC) and immunofluorescence (IF) include: cytokeratin (DAKO, Z0622, 1:200), active $\beta$-catenin (CST, \#8814, 1:1000), $\beta$ catenin (Abcam, ab6302, 1:1000), Plf (Santa Cruz, sc47347, 1:200), and Pcdh12 (MAB7926, 1:200).

\section{In situ hybridization}

In situ hybridization with isotopes-labeled antisense RNA probes was performed on cryosections $(10 \mu \mathrm{m})$ as 
previously described [55]. Whole-mount in situ hybridization with digoxygenin (DIG)-labeled antisense RNA probes was conducted using standard procedures. The primers for probe production are listed in Additional file 1: Table S1.

\section{Trophoblast stem cell derivation, culture, and differentiation}

Sfrp1 and Sfrp $5 \mathrm{dKO}$ as well as WT TS cells were derived from E3.5 mouse blastocysts as described previously [28]. Briefly, blastocysts were obtained from E3.5 uterus, and transferred to four-well tissue culture dish containing the mitomycin C-treated MEF feeders in TS medium+F4H [RPMI 1640 (Gibco, 31870082) containing 20\% FBS (Gibco, 16000044), $1 \mathrm{mM}$ sodium pyruvate, $100 \mu \mathrm{M} \beta$-mercaptoethanol, 2 mM L-glutamine, 25 ng/ml FGF4 (Peprotech, 100-31), and 1 $\mathrm{mg} / \mathrm{ml} \mathrm{Heparin} \mathrm{(Sigma,} \mathrm{2,608,411)]} \mathrm{and} \mathrm{cultured} \mathrm{at} 37^{\circ} \mathrm{C}, 5 \%$ $\mathrm{CO}_{2}$. Then, the blastocysts hatched from zona pellucida and attached to the wells to form outgrowth. After disaggregation of the blastocyst outgrowth, feed cells with 70\%FCM + $1.5 x \mathrm{~F} 4 \mathrm{H} \quad(30 \%$ TS medium, $70 \%$ mouse fibroblastconditioned medium, 1.5x FGF4/Heparin) for early passages and with $70 \% \mathrm{FCM}+1 \mathrm{xF} 4 \mathrm{H}$ for maintenance. For TS cells differentiation, TS cell medium was used without the supplementation of FGF4, heparin, and FCM. For the treatment of TSC, CHIR99021 (Biovision, 1748-5), XAV-939 (MedChemExpress, HY-15147), and iCRT3 (MedChemExpress, HY103705) were used.

\section{Quantitative real-time PCR}

RNA extraction and quantitative RT-PCR was performed as described [9]. Briefly, cells were directly lysed in RNAiso plus (TAKARA, 9109) after pumping out the medium. RNA was extracted in the upper layer after chloroform added and centrifuged. Purified RNA could be obtained after precipitation with isopropanol and washing with ethanol. cDNA was reverse transcripted with PrimeScript ${ }^{\mathrm{TN}} \mathrm{RT}$ reagent Kit with gDNA Eraser (Perfect Real Time) (TAKA RA, RR047A) according to the manufacturer's instructions. qRT-PCR were performed with TB Green ${ }^{\circledR}$ Premix Ex Taq ${ }^{\text {TM }}$ II (Tli RNaseH Plus) (TAKARA, RR820A). All assays were performed at least three times. The primers for real-time PCR are all listed in Additional file 1: Table S1.

\section{Western blotting}

Protein extraction and Western blotting were performed as described previously [9]. Antibodies used for western blotting include active $\beta$-catenin (CST, \#8814, 1:1000), $\beta$-catenin (Abcam, ab6302, 1:1000), and $\beta$-actin (Bioworld, AP0063, 1:5000).

\section{RNA-seq and data analysis}

RNA from Cyp19-cre and Cyp19-cre; Ctnnb1 $1^{f(E X 3) /+}$ placentas at E8.5 were extracted using RNeasy Micro Kit
(QIAGEN, 74004) according to the manufacturer's instructions. Purified RNA was prepared using TruSeq ${ }^{\circ}$ RNA Sample Preparation V2 (Illumina, RS-122-2001) and subjected to 50-bp single-end sequencing with a BGISEQ-500 sequencer. RNA-seq raw data were initially filtered to obtain clean data after quality control. Clean data were aligned to the mouse genome (mm10) by HISAT2. Raw counts for each gene were calculated by EdgeR. DEGs were defined as genes with $P$ value less than 0.05 and fold change larger than 1.5.

\section{ChIP-Seq and ChIP-qPCR}

ChIP was performed according to the reported previously [56]. Briefly, about $2 \times 10^{6}$ TS cells were cross-linked with $16 \%$ formaldehyde (Cell Signaling Technology, 12,606) at final concentration of $1 \%$ at room temperature for $10 \mathrm{~min}$ and quenched with $1 / 10$ volume of $1.25 \mathrm{M}$ glycine for 15 min on ice. Cell lysate in lysis buffer III were sonicated using Bioruptor pico (Diagenode) and then incubated with $4 \mu \mathrm{g}$ non-phospho (active) $\beta$-catenin antibody (Cell Signaling Technology, 8814) overnight at $4^{\circ} \mathrm{Cwith}$ rotation. Immunoprecipitated complexes were collected with $15 \mu \mathrm{l}$ Protein A Dynabeads (Invitrogen, 10006D) for $1 \mathrm{~h}$ at $4{ }^{\circ} \mathrm{C}$ with rotation. Subsequently, beads were washed sequentially once with low-salt buffer, twice with high-salt buffer, once with $\mathrm{LiCl}$ buffer, twice with $\mathrm{TE}$, and then eluted in $400 \mu \mathrm{l}$ of elution buffer for $30 \mathrm{~min}$ at $65^{\circ} \mathrm{C}$ with shaking. The eluates were incubation at $65^{\circ} \mathrm{C}$ for $8 \mathrm{~h}$ to reverse the cross-linking. Next, eluates were treated with proteinase $\mathrm{K}$ for $1 \mathrm{~h}$ at $55^{\circ} \mathrm{C}$ and then RNase A for $30 \mathrm{~min}$ at $37^{\circ} \mathrm{C}$ before DNA was extracted and purified. The ChIP libraries were prepared according to the instruction manual using KAPA DNA HyperPrep Kits (Roche, KK8502) and then run on the Illumina sequencer Hiseq-Xten PE150. Primers for qPCR were listed in Additional file 1: Table S1.

\section{ChIP-Seq analysis}

The primary analysis of ChIP-Seq datasets were performed by using Illumina's Genome Analysis pipeline. The sequencing reads were aligned to the mouse genome (mm10) by HISAT2. Only uniquely aligned reads were kept. MACS2 was applied for peak call using default parameters.

\section{Statistical analysis}

Statistical analysis was performed with the GraphPad Prism 8 software. In data that was normally distributed, Student's $t$ test was performed to determine the significance of a difference between two groups. When comparing the means of more than two groups, a one-way ANOVA was used. Data were presented as means \pm SEM in all experiments unless otherwise indicated. ${ }^{*} P<$ 0.05 was considered to indicate a significant result. 


\section{Supplementary information}

Supplementary information accompanies this paper at https://doi.org/10. 1186/s12915-020-00883-4.

Additional file 1: : Figure S1. The expression of Sfrp1, Sfrp2 and Sfrp5 during early placental development. A The expression of Sfrp1, Sfrp2, and Sfrp5 was analyzed by whole-mount in situ hybridization at E7.5 and E8.5. B The expression of Sfrp 1 and Sfrp 5 was detected by in situ hybridization at E7.5-E9.5. The signals were pink. Images in (A) and (B) are representatives of at least two independent experiments. Figure S2. Statistical description of the phenotype of the Sfrp1 and Sfrp5 dKO placenta. A Statistical analysis of spongiotrophoblast thickness and TGCs number in WT and dKO placenta on E9.5. B Quantification of active- $\beta$-catenin signal intensity in Fig. 2a. ${ }^{*}, P<0.05$. Figure S3. Nuclear localization of active- $\beta$ catenin increased in the placenta of $\mathrm{Ctnnb}^{\mathrm{\Delta} 1^{\alpha+}}$ conceptus. A Immunohistochemistry of active- $\beta$-catenin in $C \operatorname{Cnnb} 1^{f(E X 3) /+}$ and $C t_{n n b} 1^{\Delta /+}$ placenta on E9.5. B Quantification of active- $\beta$-catenin signal intensity in (A). ${ }^{*}, P<$ 0.05. Figure S4. Global stabilization of $\beta$-catenin leads to impaired trophoblast development and embryonic lethality. HE and CK staining of the sections of $C t_{n n b} f^{f(E X 3) /+}$ and $C t_{n n b} 1^{\Delta+}$ conceptus on E7.5. Images are representatives of at least three independent experiments. Dec, decidua; EPC, ectoplacental cone; Em, embryo; TGC, trophoblast giant cell. Figure S5. Trophoblast-specific stabilization of $\beta$-catenin induces hyperactivation of canonical Wnt pathway. A The localization of active- $\beta$ catenin was revealed by immunostaining at E7.5. Cy3-labeled active $\beta$ catenin in red, DAPI-labeled nuclei in blue. Images are representatives of at least three independent experiments. B Quantification of active- $\beta$ catenin signal intensity in $(A) .{ }^{*}, P<0.05$. Figure $\mathbf{S 6}$. Decreased genes with trophoblast-specific stabilization of $\beta$-catenin are related to cell cycles. KEGG analysis of the decreased genes (A, B) between Cyp 19-cre and Cyp 19-cre; Ctnnb $1^{f(E \times 3) /+}$ placentas (Fold change $>1.5, P$ value $\left.<0.05\right)$. Figure S7. Sfrp1 and Sfrp5 deficiency led to increased activity of Wnt signaling. A TOP-Flash assay in the WT and dKO TS cells. B The expression of Ascl2, Tpbpa and P/1 in dKO TS cells differentiated for 4 days, in the presence of XAV-939 or not. * ${ }^{*} P<0.05$. C QRT-PCR analysis of the expression of Ascl2, Tpbpa and P/1 in dKO TS cells differentiated for 4 days, with Ascl2 overexpression or not. Figure S8. TCF/LEF might be not required for $\beta$ catenin-mediated Ascl2 suppression. A The expression of $\mathrm{Ascl} 2$ in WT TS cells treated with indicated conditions. iCRT3, a small molecule that abrogates $\beta$-catenin-TCF interaction. * ${ }^{*} P<0.05$. ns, not significant. B The top 13 conserved transcription factors binding motif at the $\beta$-catenin binding site, $20 \mathrm{~kb}$ upstream of the Ascl2 gene. Table S1. Primers Information.

Additional file 2: : Table S2. Differentially expressed genes (DEG) between the Cyp19-cre and Cyp19-Cre; Ctnnb1 $1^{f(E \times 3) /+}$ placentas (fold change > 1.5). Table S3. Genes expressed in the Cyp19-cre and Cyp19cre; Ctnnb $1^{f((E \times 3) /+}$ placentas.

\section{Abbreviations}

Ascl2: achaete-scute complex homolog-like 2 (also known as Mash2); EPC: Ectoplacental cone; ExE: Extraembryonic ectoderm; Gcm 1: Glial cells missing-1; Hand1: Heart and neural crest derivatives expressed transcript 1; TGC: Trophoblast giant cell

\section{Acknowledgements}

We are grateful to Drs. Makoto Mark Taketo, Akihiko Shimono, and Gustavo

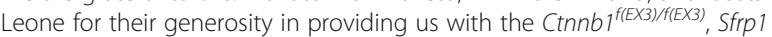
and Sfrp5 double mutant, and Cyp19-cre mice, respectively.

\section{Authors' contributions}

L.J., W.Q., and W.H. designed the research; B.H., L.D., X.Y., S.Y., M.C., Y.Y., W.C., H.Q., L.S., and C.H. performed the research; L.F., D.W., C.B., and K.S. analyzed the data; L.J., W.Q., B.H., and W.H. wrote the paper. All authors read and approved the final manuscript.

\section{Funding}

This work was supported in parts by National Key R\&D program of China (2017YFC1001402 to H.W., 2018YFC1004102 to J.L., 2018 YFC1004401 to S.K., and 2018YFC1004404 to B.C.), National Natural Science Foundation of China (81830045 to H.W., 31971071 and 81741035 to J.L.), and the Fundamental Research Funds for the Central Universities (20720180041 J.L. and
20720180039 to S.K., 20720190073 to W.D). The funders had no role in study design, data collection and analysis, decision to publish, or preparation of the manuscript.

\section{Availability of data and materials}

All sequencing data including RNA-seq and ChIP-seq data have been deposited in the Gene Expression Omnibus (GEO) database with the accession codes GSE146433 (https://www.ncbi.nlm.nih.gov/geo/query/acc.cgi?acc= GSE146433) and GSE146432 (https://www.ncbi.nlm.nih.gov/geo/query/acc. cgi?acc=GSE146432) [57], respectively. If any additional information and materials are needed, they will be available upon request from the corresponding authors.

\section{Ethics approval and consent to participate}

Mice were housed in the Animal Care Facility of Xiamen University, and animal experiments mentioned in the manuscript have been conducted according to the guidelines for the care and use of laboratory animals.

\section{Consent for publication}

Not applicable

\section{Competing interests}

The authors declare that they have no competing interests.

\section{Author details}

${ }^{1}$ Reproductive Medical Center, The First Affiliated Hospital of Xiamen University, Xiamen 361003, Fujian, People's Republic of China. ${ }^{2}$ Fujian Provincial Key Laboratory of Reproductive Health Research, School of Medicine, Xiamen University, Xiamen 361102, Fujian, People's Republic of China. ${ }^{3}$ State Key Laboratory of Stem Cell and Reproductive Biology, Institute of Zoology, Chinese Academy of Sciences, Beijing 100101, People's Republic of China. ${ }^{4}$ Department of Surgery, The Ohio State University Wexner Medical Center, Ohio 43210 Columbus, USA.

Received: 17 April 2020 Accepted: 29 September 2020

Published online: 27 October 2020

\section{References}

1. Rossant J, Cross JC. Placental development: lessons from mouse mutants. Nat Rev Genet. 2001;2(7):538-48.

2. Maltepe E, Bakardjiev Al, Fisher SJ. The placenta: transcriptional, epigenetic, and physiological integration during development. J Clin Invest. 2010;120(4) 1016-25.

3. Latos PA, Hemberger M. From the stem of the placental tree: trophoblast stem cells and their progeny. Development. 2016;143(20):3650-60.

4. Hemberger M, Hanna CW, Dean W. Mechanisms of early placental development in mouse and humans. Nat Rev Genet. 2020;21(1):27-43.

5. Guillemot F, Nagy A, Auerbach A, Rossant J, Joyner AL. Essential role of Mash-2 in extraembryonic development. Nature. 1994;371(6495):333-6.

6. Jiang B, Kamat A, Mendelson CR. Hypoxia prevents induction of aromatase expression in human trophoblast cells in culture: potential inhibitory role of the hypoxia-inducible transcription factor Mash-2 (mammalian achaetescute homologous protein-2). Mol Endocrinol. 2000;14(10):1661-73.

7. Wakeland AK, Soncin F, Moretto-Zita M, Chang CW, Horii M, Pizzo D, et al. Hypoxia directs human extravillous trophoblast differentiation in a hypoxiainducible factor-dependent manner. Am J Pathol. 2017;187(4):767-80.

8. Knofler M, Pollheimer J. Human placental trophoblast invasion and differentiation: a particular focus on Wnt signaling. Front Genet. 2013;4:190.

9. Lu J, Zhang S, Nakano H, Simmons DG, Wang S, Kong S, et al. A positive feedback loop involving Gcm1 and Fzd5 directs chorionic branching morphogenesis in the placenta. PLoS Biol. 2013;11(4):e1001536.

10. Parr BA, Cornish VA, Cybulsky MI, McMahon AP. Wnt7b regulates placental development in mice. Dev Biol. 2001;237(2):324-32.

11. Monkley SJ, Delaney SJ, Pennisi DJ, Christiansen JH, Wainwright BJ. Targeted disruption of the Wnt2 gene results in placentation defects. Development. 1996:122(11):3343-53.

12. Ishikawa T, Tamai Y, Zorn AM, Yoshida H, Seldin MF, Nishikawa S, et al Mouse Wnt receptor gene Fzd5 is essential for yolk sac and placental angiogenesis. Development. 2001;128(1):25-33. 
13. Galceran J, Farinas I, Depew MJ, Clevers H, Grosschedl R. Wnt3a-/--like phenotype and limb deficiency in Lef1(-/-)Tcf1(-/-) mice. Genes Dev. 1999; 13(6):709-17.

14. Aoki M, Mieda M, Ikeda T, Hamada Y, Nakamura H, Okamoto H. R-spondin3 is required for mouse placental development. Dev Biol. 2007;301(1):218-26.

15. Matsuura K, Jigami T, Taniue K, Morishita Y, Adachi S, Senda T, et al. Identification of a link between Wnt/beta-catenin signalling and the cell fusion pathway. Nat Commun. 2011;2:548.

16. Haider S, Meinhardt G, Saleh L, Kunihs V, Gamperl M, Kaindl U, et al. Selfrenewing trophoblast organoids recapitulate the developmental program of the early human placenta. Stem Cell Reports. 2018;11(2):537-51.

17. Okae H, Toh H, Sato T, Hiura H, Takahashi S, Shirane K, et al. Derivation of human trophoblast stem cells. Cell Stem Cell. 2018;22(1):50-63 e6.

18. Turco MY, Gardner L, Kay RG, Hamilton RS, Prater M, Hollinshead MS, et al. Trophoblast organoids as a model for maternal-fetal interactions during human placentation. Nature. 2018;564(7735):263-7.

19. Pollheimer J, Loregger T, Sonderegger S, Saleh L, Bauer S, Bilban M, et al. Activation of the canonical wingless/T-cell factor signaling pathway promotes invasive differentiation of human trophoblast. Am J Pathol. 2006;168(4):1134-47.

20. Kawano Y, Kypta R. Secreted antagonists of the Wnt signalling pathway. J Cell Sci. 2003;116(Pt 13):2627-34.

21. Liang CJ, Wang ZW, Chang YW, Lee KC, Lin WH, Lee JL. SFRPS are biphasic modulators of Wnt-signaling-elicited cancer stem cell properties beyond extracellular control. Cell Rep. 2019;28(6):1511-25 e5.

22. Hemberger M, Cross JC. Genes governing placental development. Trends Endocrinol Metab. 2001;12(4):162-8.

23. Firulli $A B$, McFadden DG, Lin $Q$, Srivastava D, Olson EN. Heart and extraembryonic mesodermal defects in mouse embryos lacking the bHLH transcription factor Hand1. Nat Genet. 1998;18(3):266-70.

24. Riley P, Anson-Cartwright L, Cross JC. The Hand1 bHLH transcription factor is essential for placentation and cardiac morphogenesis. Nat Genet. 1998; 18(3):271-5.

25. Harada N, Tamai Y, Ishikawa T, Sauer B, Takaku K, Oshima M, et al. Intestinal polyposis in mice with a dominant stable mutation of the beta-catenin gene. EMBO J. 1999;18(21):5931-42.

26. Jubb AM, Chalasani S, Frantz GD, Smits R, Grabsch HI, Kavi V, et al. Achaetescute like 2 (ascl2) is a target of Wnt signalling and is upregulated in intestinal neoplasia. Oncogene. 2006;25(24):3445-57.

27. Hatzis $P$, van der Flier LG, van Driel MA, Guryev V, Nielsen F, Denissov S, et al. Genome-wide pattern of TCF7L2/TCF4 chromatin occupancy in colorectal cancer cells. Mol Cell Biol. 2008;28(8):2732-44.

28. Tanaka S, Kunath T, Hadjantonakis AK, Nagy A, Rossant J. Promotion of trophoblast stem cell proliferation by FGF4. Science. 1998;282(5396):2072-5.

29. Li C, Zhang S, Lu Y, Zhang Y, Wang E, Cui Z. The roles of Notch3 on the cell proliferation and apoptosis induced by CHIR99021 in NSCLC cell lines: a functional link between Wnt and Notch signaling pathways. PLoS One. 2013:8(12):e84659.

30. Giakountis A, Moulos P, Zarkou V, Oikonomou C, Harokopos V, Hatzigeorgiou AG, et al. A positive regulatory loop between a Wntregulated non-coding RNA and ASCL2 controls intestinal stem cell fate. Cell Rep. 2016;15(12):2588-96.

31. Estaras C, Benner C, Jones KA. SMADs and YAP compete to control elongation of beta-catenin: LEF-1-recruited RNAPII during hESC differentiation. Mol Cell. 2015;58(5):780-93.

32. Clevers H, Nusse R. Wnt/beta-catenin signaling and disease. Cell. 2012; 149(6):1192-205

33. Clevers H, Loh KM, Nusse R. Stem cell signaling. An integral program for tissue renewal and regeneration: Wnt signaling and stem cell control. Science. 2014;346(6205):1248012.

34. Steinhart Z, and Angers S. Wnt signaling in development and tissue homeostasis. Development. 2018;145(11):146589.

35. Ng LF, Kaur P, Bunnag N, Suresh J, Sung ICH, Tan QH, et al. WNT Signaling in Disease. Cells. 2019;8(8):8080826.

36. Logan CY, Nusse R. The Wnt signaling pathway in development and disease. Annu Rev Cell Dev Biol. 2004;20:781-810.

37. Korinek V, Barker N, Morin PJ, van Wichen D, de Weger R, Kinzler KW, et al. Constitutive transcriptional activation by a beta-catenin-Tcf complex in APC-/- colon carcinoma. Science. 1997;275(5307):1784-7.

38. Kinzler KW, Nilbert MC, Su LK, Vogelstein B, Bryan TM, Levy DB, et al. Identification of FAP locus genes from chromosome 5q21. Science. 1991; 253(5020):661-5
39. Nishisho I, Nakamura Y, Miyoshi Y, Miki Y, Ando H, Horii A, et al. Mutations of chromosome 5 q21 genes in FAP and colorectal cancer patients. Science. 1991;253(5020):665-9.

40. Novakovic B, Rakyan V, Ng HK, Manuelpillai U, Dewi C, Wong NC, et al. Specific tumour-associated methylation in normal human term placenta and first-trimester cytotrophoblasts. Mol Hum Reprod. 2008;14(9):547-54.

41. Wong NC, Novakovic B, Weinrich B, Dewi C, Andronikos R, Sibson M, et al. Methylation of the adenomatous polyposis coli (APC) gene in human placenta and hypermethylation in choriocarcinoma cells. Cancer Lett. 2008; 268(1):56-62.

42. Meinhardt G, Haider S, Haslinger P, Proestling K, Fiala C, Pollheimer J, et al. Wnt-dependent T-cell factor-4 controls human etravillous trophoblast motility. Endocrinology. 2014;155(5):1908-20.

43. Guillemot F, Caspary T, Tilghman SM, Copeland NG, Gilbert DJ, Jenkins NA, et al. Genomic imprinting of Mash2, a mouse gene required for trophoblast development. Nat Genet. 1995;9(3):235-42.

44. He TC, Sparks AB, Rago C, Hermeking H, Zawel L, da Costa LT, et al. Identification of C-MYC as a target of the APC pathway. Science. 1998; 281(5382):1509-12.

45. Yan D, Wiesmann M, Rohan M, Chan V, Jefferson AB, Guo L, et al. Elevated expression of axin2 and hnkd mRNA provides evidence that Wnt/beta -catenin signaling is activated in human colon tumors. Proc Natl Acad Sci U S A. 2001;98(26):14973-8.

46. Smartt HJ, Greenhough A, Ordonez-Moran P, Talero E, Cherry CA, Wallam $C A$, et al. beta-catenin represses expression of the tumour suppressor 15prostaglandin dehydrogenase in the normal intestinal epithelium and colorectal tumour cells. Gut. 2012;61(9):1306-14.

47. Spencer GJ, Utting JC, Etheridge SL, Arnett TR, Genever PG. Wnt signalling in osteoblasts regulates expression of the receptor activator of NFkappaB ligand and inhibits osteoclastogenesis in vitro. J Cell Sci. 2006;119(Pt 7):1283-96.

48. Jamora C, DasGupta R, Kocieniewski P, Fuchs E. Links between signal transduction, transcription and adhesion in epithelial bud development. Nature. 2003;422(6929):317-22.

49. Anson-Cartwright L, Dawson K, Holmyard D, Fisher SJ, Lazzarini RA, Cross JC. The glial cells missing-1 protein is essential for branching morphogenesis in the chorioallantoic placenta. Nat Genet. 2000;25(3):311-4.

50. Oh-McGinnis R, Bogutz AB, Lefebvre L. Partial loss of Ascl2 function affects all three layers of the mature placenta and causes intrauterine growth restriction. Dev Biol. 2011;351(2):277-86.

51. Tanaka M, Gertsenstein M, Rossant J, Nagy A. Mash2 acts cell autonomously in mouse spongiotrophoblast development. Dev Biol. 1997;190(1):55-65.

52. Wenzel PL, Leone G. Expression of Cre recombinase in early diploid trophoblast cells of the mouse placenta. Genesis. 2007:45(3):129-34

53. Satoh W, Gotoh T, Tsunematsu Y, Aizawa S, Shimono A. Sfrp1 and Sfrp2 regulate anteroposterior axis elongation and somite segmentation during mouse embryogenesis. Development. 2006;133(6):989-99.

54. Satoh W, Matsuyama M, Takemura H, Aizawa S, Shimono A. Sfrp1, Sfrp2, and Sfrp5 regulate the Wnt/beta-catenin and the planar cell polarity pathways during early trunk formation in mouse. Genesis. 2008;46(2):92-103.

55. Wang Q, Lu J, Zhang S, Wang S, Wang W, Wang B, et al. Wnt6 is essential for stromal cell proliferation during decidualization in mice. Biol Reprod. 2013;88(1):5

56. Lee TI, Johnstone SE, Young RA. Chromatin immunoprecipitation and microarray-based analysis of protein location. Nat Protoc. 2006;1(2):729-48.

57. Bao H, Liu D, Xu Y, Sun Y, Mu C, Yu Y, et al. Hyperactivated Wnt- $\beta$-catenin signaling in the absence of sFRP1 and sFRP5 represses Ascl2 expression derailing normal spongiotrophoblast differentiation. NCBI accession number GSE146433 and GSE146432. 2020. https://www.ncbi.nlm.nih.gov/geo/query/ acc.cgi?acc=GSE146433; https://www.ncbi.n/m.nih.gov/geo/query/acc. cgi?acc=GSE146432. Accessed 5 June 2020.

\section{Publisher's Note}

Springer Nature remains neutral with regard to jurisdictional claims in published maps and institutional affiliations. 